\title{
Prevalencia de discapacidad física en adultos mayores de provincias seleccionadas, Cuba 2000-2004
}

\author{
Prevalence of physical disability in elders from selected \\ provinces, Cuba 2000-2004
}

Héctor D. Bayarre Vea'; J ulia S. Pérez Piñero'; J esús Menéndez J iménez"; Teddy O. Tamargo Barbeito'"'; Agustín Morejón Carralero'v; Dayamí Garrido Díaz"; Arlín González de Piñera Marrero'v

'Dr. C. de la Salud. Escuela Nacional de Salud Pública. La Habana, Cuba.

"Máster en Gerontología Médica y Social. Centro de Investigaciones sobre longevidad, envejecimiento y salud. La Habana, Cuba.

"'Máster en Salud Pública. Escuela Nacional de Salud Pública. La Habana, Cuba.

IVEspecialista de I Grado en Bioestadística. Escuela Nacional de Salud Pública. La Habana, Cuba.

VEspecialista de II Grado en Bioestadística. Escuela Nacional de Salud Pública. La Habana, Cuba.

\section{RESUMEN}

Introducción La discapacidad física es uno de los problemas más importantes que enfrenta la comunidad científica cubana.

Objetivos Estimar la prevalencia de discapacidad física en adultos mayores de Ciudad de La Habana, Las Tunas, Granma, Holguín y Camagüey, entre los años 2000 y 2004.

Métodos Se realizó un estudio descriptivo sobre muestras complejas de adultos mayores seleccionados de forma independiente para cada provincia. Se aplicó el Índice Lawton y el Examen Mínimo del Estado Mental. Se estimaron tasas de prevalencia de discapacidad física, globales y específicas.

Resultados La discapacidad física osciló entre 13,9\% (Holguín) y 36,6 \%

(Granma). Se incrementó con la edad, desde 4,5\%, para las personas de 60-69 años que residen en Holguín, hasta 83 \% en los de 80 y más de Las Tunas; 
predominó en mujeres de todas las provincias, con tasas que alcanzaron el $40 \%$, excepto para Holguín. Los ancianos de esta provincia tuvieron menor afectación que los de las restantes provincias en todas las actividades, excepto en el manejo de la casa, de menor afectación en los de Las Tunas (10,9\%). El uso del teléfono fue lo más conservado independientemente de la provincia, mientras que la responsabilidad con los medicamentos mostró valores parecidos en cada una de éstas.

Conclusiones La discapacidad física es frecuente en la población investigada, con excepción de quienes residen en Holguín; se incrementa con la edad, predomina en el sexo femenino y se genera por pérdida de actividades que demandan mayor desplazamiento. Las variables psicosociales más afectadas son: el analfabetismo y la pérdida de rol social. En Holguín predomina la sensación de inactividad y la insatisfacción con las actividades cotidianas, la primera de ellas presente además, en Las Tunas y la segunda en Camagüey. En Ciudad de La Habana y Granma se adiciona el sentimiento de soledad.

Palabras clave: Adulto mayor, discapacidad física, prevalencia, provincias, Cuba.

\section{ABSTRACT}

Introduction Physical disability is one of the most important problems that the Cuban scientific community faces.

Objective To estimate the prevalence of physical disability in elders from the City of Havana, Las Tunas, Gramma, Holguín and Camagüey provinces from 2000 to 2004. Methods A descriptive study of complex samples of randomly selected elders from each of the above provinces was conducted. Lawton index and Minimal Mental Status Examination were applied. Global and specific physical disability prevalence rates were estimated.

Results Physical disability ranged from $13.9 \%$ (Holguín province) to $36.6 \%$ (Gramma province). It increased with the age, from $4.5 \%$ in 60-69 age group dwelling in Holguín to $83 \%$ in 80 years and over group in Las Tunas and it was predominant in females in the five provinces, with rates of $40 \%$, except for Holguín. Elders in this last province were less disabled than those of the other provinces in all the activities except for housekeeping in which older people from Las Tunas were more capable (10.9\%). Use of telephone was the most preserved ability in elders regardless of the province whereas the responsibility for taking their medicines showed similar values.

Conclusions Physical disability is common in the researched older population except for that of Holguín province; it increases with the age, is predominant in females and derives from loss of mobility activities. The most affected psychosocial variables are illiteracy and lack of active social role. Feelings of idleness and dissatisfaction with daily life activities predominated in the elderly from Holguín; the former was also present in Las Tunas province and the latter in Camagüey province. Additionally, the feeling of loneliness also prevailed in the City of Havana and Gramma provinces.

Key words: Older people, physical disability, prevalence, provinces, Cuba. 


\section{NTRODUCCI ÓN}

La prolongación de la esperanza de vida ha sido un anhelo de la humanidad. En este sentido, el envejecimiento puede ser considerado un logro en el que mucho ha aportado el desarrollo científico técnico alcanzado. ${ }^{1}$ Sin embargo, la longevidad está produciendo desafíos sin precedentes a los responsables de la elaboración de políticas en general y a los ciudadanos en particular, pues se deberán mantener los niveles de seguridad social y económica, y la oferta de servicios de salud de calidad a un segmento de la población que, por su avanzada edad, se encuentra en desventaja para afrontar las exigencias sociales de la contemporaneidad. ${ }^{2}$

Las discapacidades físicas y mentales, íntimamente relacionadas con el incremento de la expectativa de vida, se consideran entre los problemas más graves a enfrentar en la actualidad. ${ }^{3}$ Su evaluación es difícil, ya que estas son consecuencia de interacciones entre alteraciones estructurales y funcionales del organismo, unido a una multitud de factores psicológicos y sociales.

La Organización Mundial de la Salud (OMS), considera que las Actividades de la Vida Diaria (AVD), constituyen el indicador más importante para medir el funcionamiento en el anciano. ${ }^{1}$ Varias son las definiciones que a las AVD se les ha dado. Gallagher y Thompson las definen como la capacidad del individuo para llevar a cabo las actividades cotidianas esenciales, Fillembaun ${ }^{4}$ como la capacidad de valerse por sí mismo y desarrollar las actividades propias de la vida diaria. Patterson y Eberly ${ }^{5}$ como un conjunto de conductas, cuya ejecución regular y apropiada es necesaria para el cuidado personal y el mantenimiento de una vida independiente.

Las AVD se dividen, de manera general, en básicas (AVDB) e instrumentadas (AIVD). Las AVDB son aquellas habilidades esenciales para llevar a cabo las actividades de autocuidado: alimentación, vestirse, aseo personal, necesidades fisiológicas y otras, mientras que las AIVD son aquellas más complejas y necesarias para desarrollar una vida independendiente: uso del teléfono, realización de compras, preparación de comidas, mantenimiento de la casa, lavado de la ropa, uso del transporte público, autoadministración de medicamentos y capacidad para manejar las finanzas. ${ }^{6}$

Para medir las AVD se ha utilizado diferentes instrumentos, dentro de los que se destacan por su uso, el Índice de Katz (IK) para las AVDB, confeccionado en $1963^{7}$ y el Índice de Lawton (IL) para las AIVD, que data de $1969 .{ }^{6}$ Este último introduce un nivel más complejo en la evaluación, ya que propicia que se puedan medir aspectos que van más allá del autocuidado.

Las investigaciones que abordan el tema de discapacidad física en la tercera edad, medida a través de las AVD son de aparición relativamente reciente. Inkster en 1977, Patterson y Eberly en 1982, estudiaron las AVD en pacientes rehabilitados, con la finalidad principal de identificar déficit en habilidades específicas para la vida diaria, que proporcionen la base para definir las conductas. ${ }^{1}$

Desde la década del 80 se han realizado varios estudios de prevalencia de la discapacidad física en el anciano, entre ellos se destacan los de Bond y otros en 1982, Fillembaun en 1984, Pearlman y Utilman en 1988 y Pardavilla en 1989. En ellos resalta la heterogeneidad en las definiciones de discapacidad física utilizadas, la que es considerada por algunos, si el sujeto está incapacitado para realizar al menos una actividad instrumentada de la vida diaria (una AIVD), en tanto que otros señalan como discapacitados físicos los imposibilitados para la realización de dos 
AIVD. Un tercer grupo de autores fija tres AIVD como número mínimo de afectaciones para clasificar a un individuo en el grupo de discapacitados. ${ }^{4,7-10}$

Varios son los estudios que sobre la discapacidad física en el anciano, se han realizado en Cuba. En uno se intentó caracterizar la población anciana residente en el área de salud del Policlínico "Santiago de las Vegas", en el Municipio Boyeros, en relación con un grupo de presuntos factores de riesgo. ${ }^{11}$ En otro, se estudiaron algunos factores biológicos y sociales que supuestamente influyen sobre el estado de salud de la población anciana de un área de salud del municipio Playa. ${ }^{1}$ Más recientemente, entre 1995 y 1999, un grupo de investigadores de la Escuela Nacional de Salud Pública (ENSAP) y del Centro I beroamericano para la Tercera Edad (CITED) realizó varias investigaciones sobre este objeto de estudio en el ámbito comunitario. Así, se estimó la prevalencia de discapacidad física y mental, y los factores de riesgo de discapacidad física en ancianos del municipio Playa, ${ }^{1}$ además, se efectuaron dos investigaciones en municipios de la provincia Las Tunas. ${ }^{12}$ Estos estudios han permitido estimar la prevalencia de las discapacidades físicas, que se encuentra entre el 24,9 y 45,3 por ciento.

A pesar de la información brindada por los estudios antes mencionados, es menester continuar realizando investigaciones en otros contextos del país y en los niveles provincial y nacional, con vistas a brindar datos útiles para la gestión en salud en estos niveles, al constituir evidencia científica actualizada sobre la magnitud de la problemática, las principales variables relacionadas con su aparición y su comportamiento temporal.

Teniendo en cuenta los argumentos anteriores, se decidió realizar esta investigación, con el propósito de aumentar el caudal de conocimientos acerca de la prevalencia de las discapacidades física en las personas de edad avanzada que aporte elementos para el diseño de estrategias de intervención a escala individual, familiar y comunitaria en la población de personas de edad de Ciudad de La Habana, Camagüey, Las Tunas, Granma y Holguín. Además, brindará información válida para la planificación de recursos y servicios, por lo que se espera que influya sobre la organización de los servicios, la dirección y el control, así como sobre la aplicación de técnicas gerenciales, entre ellas, la toma de decisiones y el establecimiento de prioridades. Esto sería posible si con el estudio se logra responder la siguiente interrogante:

¿Cuál es la magnitud de la discapacidad física global según variables seleccionadas en la población anciana de Ciudad de La Habana, Camagüey, Las Tunas, Granma y Holguín?

Para dar respuesta a la interrogante planteada se formuló el siguiente objetivo:

Estimar la prevalencia de discapacidad física en los adultos mayores de las provincias Ciudad de La Habana, Las Tunas, Granma, Holguín y Camagüey, en los años 2000, 2002 y 2004.

\section{MÉTODOS}

El trabajo es un estudio descriptivo, de corte transversal, de los conocidos estudios de prevalencia, realizado en la población de 60 años y más residente en Ciudad de La Habana, Las Tunas, Granma, Holguín y Camagüey, entre el año 2000 y el 2004. 
El estudio incluyó las variables siguientes: provincia, discapacidad física, sexo, edad, enfermedades crónicas, secuela posfractura de cadera, capacidad para usar el teléfono, realización de compras, preparación de alimentos, manejo de la casa, lavado de la ropa, modo de transporte, responsabilidad con los propios medicamentos y capacidad para manejar las finanzas.

\section{Aspectos éticos}

Antes de ser incluidos en el estudio se le solicitó a cada adulto mayor, o en su defecto a un familiar (en caso de deterioro cognitivo importante), el consentimiento para su participación. Se explicaron los objetivos del estudio y la importancia de su colaboración. Se les garantizó la confidencialidad de la información que ellos aportarían y la posibilidad de abandonar el estudio si así lo deseasen, sin repercusión alguna ante la necesidad de atención médica posterior.

\section{Técnicas y procedimientos}

El universo del estudio estuvo constituido por todos los ancianos que residían en las provincias de Ciudad de La Habana y Las Tunas, durante el primer semestre del 2000; Granma y Holguín en el primer semestre de 2002 y Camagüey en el primer semestre de 2004. El tamaño de la muestra se determinó, en cada caso, a través de la fórmula para la determinación del tamaño mínimo necesario en estudios descriptivos. ${ }^{13}$ De Ciudad de La Habana se escogió una muestra de 728 ancianos, de Las Tunas se seleccionaron 698, de Granma 700, de Holguín 701 y de Camagüey 866.

En todas las provincias se utilizó una $P=45 \%$. Además, se fijó un $E_{0}=4,5$ y la confiabilidad a utilizar fue de $95 \%$. La prevalencia se obtuvo a partir de estudios anteriores realizados en Ciudad de La Habana. Se consideró un efecto de diseño (deff) de 1,5, por el que se multiplicó el valor de n. Se estimó una caída de la muestra de aproximadamente $5 \%$.

Para la selección definitiva de la muestra, se utilizó la técnica denominada Muestreo Estratificado Polietápico (MEP). ${ }^{13}$

Para la recogida de la información se aplicaron los siguientes instrumentos: Examen Mínimo del Estado Mental (EMEM), para evaluar la esfera mental, Índice de Lawton (IL) para evaluar la esfera física y la Encuesta de Factores de Riesgo de Discapacidad (EFRD), para evaluar factores de riesgo.

La aplicación de los instrumentos se realizó con la siguiente secuencia:

Se aplicó el EMEM, y si el anciano obtuvo una puntuación menor de 21 o 24 puntos en caso de ser analfabeto o no respectivamente, se consideró que existe deterioro cognoscitivo, en cuyo caso no se le aplicó la EFRD.

Se aplicó el IL a todos los ancianos o en su defecto (déficit cognoscitivo, sordos, mudos) a informantes o convivientes.

Se aplicó la EFRD para la recogida de la información referida a factores de riesgos biológicos, psicológicos y sociales a aquellos ancianos que no resultaron excluidos en el primer paso.

EI EMEN y el I L son instrumentos de amplio uso en la investigación gerontológica y cuentan con los atributos de fiabilidad y validez requeridos para su uso. 
La EFRD fue diseñada por especialistas del CITED, para ser aplicada a los ancianos. Se tomó una serie de factores de riesgo biológicos y psicosociales de discapacidad física de la literatura. El objetivo de su aplicación es la comprobación de la presencia o no de alguno de los factores en el anciano. Este instrumento no ha sido validado de forma exhaustiva, pero sí tiene un buen criterio de expertos (validez de contenido); debe aplicarse por un profesional de la salud, y el tiempo requerido para su aplicación es breve (aproximadamente cinco minutos).

La aplicación de los diferentes instrumentos se realizó por un grupo de estudiantes de excepcional rendimiento, por los médicos de la familia de los consultorios seleccionados que se motivaron con la investigación y por residentes de tercer año de la especialización en Bioestadística. Se realizó un adiestramiento previo a los entrevistadores que consistió en un seminario impartido por un psicólogo del CITED. Con el objetivo de asegurar la recogida de la información, se realizó un control de la calidad al $10 \%$ de las entrevistas seleccionadas al azar.

\section{Técnicas de procesamiento y análisis}

El tratamiento de los datos se realizó mediante programa estadístico computarizado. Se realizó la estimación puntual y por intervalo de la tasa global y específica de discapacidad física. Con la finalidad de controlar el efecto de la estructura poblacional por edad y sexo, se realizó la tipificación de tasas por el método directo. Se escogió como población tipo la suma de las muestras de las provincias del estudio. Luego se calcularon, para cada muestra, las discapacidades esperadas por variable; para ello, se multiplicaron las tasas específicas por la población tipo de la categoría correspondiente, en cada caso. Finalmente, se obtuvieron las tasas tipificadas al dividir la suma de las discapacidades esperadas por el total de la población tipo.

\section{RESULTADOS}

La estimación puntual de la prevalencia de discapacidad física en adultos mayores de las provincias estudiadas varió desde 13,9 hasta 36, $6 \%$, correspondiendo los valores extremos a las provincias Holguín y Granma respectivamente. Con excepción de Holguín, que presentó una cifra baja de este tipo de discapacidad, las restantes provincias tuvieron valores superiores al $30 \%$ (tabla 1 ).

Para el control de la posible influencia de las estructuras por edad y sexo de las poblaciones estudiadas sobre las tasas de discapacidad física estimadas, estas se tipificaron por el método directo (tabla 2).

Al controlar la edad se apreció una importante reducción de la discapacidad física para la provincia Ciudad de La Habana (de 34,8 a 24,6 \%); mientras que para las restantes provincias el cambio fue discreto. Ello permitió pensar que si las provincias en estudio estuviesen sometidas a la misma estructura de edades, la prevalencia de discapacidad física en Ciudad de La Habana fuese menor, aunque los resultados no pueden compararse con los de Holguín.

Con relación a las AIVD, las principales limitaciones" para los adultos mayores de Ciudad de La Habana fueron la capacidad para manejar sus finanzas y el modo de transporte $(21,0 \%$ y $20,7 \%$ respectivamente), mientras que las limitaciones en la responsabilidad con los medicamentos y la capacidad para usar el teléfono fueron las menos frecuentes. Las personas de edad de Camagüey presentaron mayores 
dificultades con el manejo de las finanzas (22,6\%) y el lavado de la ropa $(20,6 \%)$, en tanto que la capacidad para el uso del teléfono fue la actividad menos afectada. Los ancianos de Las Tunas tuvieron las principales limitaciones en la realización de compras $(26,8 \%)$ y la preparación de alimentos $(24,4 \%)$, mientras que el manejo de la casa, la capacidad en el manejo de las finanzas y en el uso del teléfono fueron las actividades menos afectadas. Las diligencias más complicadas en la población objeto de estudio de la provincia Granma fueron la realización de compras $(26,5$ $\%)$, la preparación de alimentos $(21,2 \%)$ y el modo de transporte $(20,5 \%)$, mientras que la actividad menos comprometida fue la capacidad para el uso del teléfono. Las mayores afectaciones en la población de Holguín se observaron en la realización de compras $(12,4 \%)$, el modo de transporte $(12,4 \%)$ y el lavado de la ropa $(12,0 \%)$, mientras que la capacidad para el uso del teléfono y el manejo de las finanzas así como la responsabilidad con los medicamentos resultaron ser las menos afectadas.

Un análisis global de las actividades por provincias permitió constatar que, los adultos mayores de la provincia Holguín tuvieron menor afectación que los de las restantes provincias en todas las actividades, con excepción del manejo de la casa, de menor afectación en los ancianos de Las Tunas. El uso del teléfono fue la actividad más conservada por la población investigada con independencia de la provincia en estudio, mientras que la responsabilidad con los medicamentos, además de ser una de las actividades menos afectadas en todas las provincias, mostró valores muy parecidos en cada una de estas.

En relación con la discapacidad física según grupos de edades y provincias del estudio, se apreció un incremento a medida que aumentó la edad, que se hizo más marcado en la población geriátrica de Camagüey y Las Tunas. En la primera ascendió desde $17,1 \%$, en el grupo de $60-69$, hasta $82,1 \%$, en el grupo de 80 y más; mientras que en la segunda esta varió desde $18,1 \%$, en el grupo de viejosjóvenes, hasta $83,0 \%$, en el grupo de viejos-viejos. También se apreció un incremento marcado en la provincia Holguín, aunque los niveles de este tipo de discapacidad encontrados en esta provincia, en todos los grupos, fueron inferiores a los registrados en las restantes.

El sexo femenino era el de mayor prevalencia en todas las provincias investigadas, con niveles muy parecidos entre las provincias Granma $(43,1 \%)$, Camagüey $(41,3$ $\%)$, Ciudad de La Habana $(40,4 \%)$ y Las Tunas $(39,6 \%$ ); mientras Holguín mostró un nivel de discapacidad muy inferior. Obsérvese en la tabla 2 la marcada diferencia en los niveles de discapacidad física según sexo.

La prevalencia de discapacidad física según otras variables biológicas y provincias se muestra en la tabla 3. La estimación de esta discapacidad en pacientes con secuelas posfractura de cadera exhibió niveles superiores respecto a la encontrada en los pacientes con enfermedades crónicas, aunque en general, para ambas se registraron altas tasas. Los mayores niveles de discapacidad para los pacientes con fractura de cadera, se encontraron en las provincias Holguín (100,0 \%), Las Tunas $(80,0 \%)$, Camagüey $(77,8 \%)$ y Ciudad de La Habana $(70,0 \%)$; mientras que para la presencia de enfermedades crónicas las mayores tasas se registraron en Ciudad de La Habana (41,6 \%) y Granma (40,8 \%). Ambas entidades mostraron elevados niveles de discapacidad física, sobre todo la fractura de cadera. Sin embargo, la imprecisión de los intervalos, quizás por el escaso número de ancianos con esta condición, limita el alcance de estos resultados.

La prevalencia de discapacidad física en ancianos según variables psicosociales en las provincias estudiadas mostró que en la población de Ciudad de La Habana se acentuaron los sentimientos de soledad con $64,1 \%(61,2-67,0 \%)$, seguidos por la 
pérdida de rol social, con $61,2 \%(56,8-65,6 \%)$. En la población Camagüey predominó la insatisfacción con actividades cotidianas $63,6 \%(58,1-69,1 \%)$ y la pérdida de rol social 59,0 \% (43,6-74,4\%). En tanto que para Las Tunas, se destacó la sensación de inactividad con una tasa de 69,8 \% (50,5-89,1 \%), seguida por la pérdida de rol social con 56,3\% (21,8-90,9\%). En Granma las variables psicosociales más afectadas fueron la pérdida de rol social con una tasa de 63,6 \% $(55,6-71,5 \%)$ y los sentimientos de soledad con $56,3 \%(39,1-76,4 \%)$, mientras que en Holguín las mayores afectaciones se relacionaron con la insatisfacción con actividades cotidianas con tasa de $50,0 \%(45,9-54,1 \%)$ y la sensación de inactividad con tasa de 50,0 \% (42,5-57,5\%).

Nótese que en general, con excepción de la provincia Holguín, se registraron tasas elevadas en virtud de las variables estudiadas y que la pérdida del rol social fue la más afectada. Además, es necesario señalar que estas variables o presuntos factores de riesgo no se pueden ver aisladamente, porque cada adulto mayor puede presentar uno o más o estar interrelacionados entre sí.

La prevalencia de discapacidad física disminuyó en la medida que los ancianos de las provincias investigadas tenían mayor escolaridad. Así, en los analfabetos de Ciudad de La Habana la tasa fue de $69,7 \%(53,8-85,6 \%)$ en tanto que para los universitarios fue de $9,1 \%(0,0-31,1 \%)$. En Camagüey la tasa de discapacidad para los analfabetos fue de $55,1 \%(4,2-69,0 \%)$ y en los universitarios de $26,2 \%$ $(15,2-37,2 \%)$. En el grupo de analfabetos de Las Tunas fue de 88,0 \% (72,1-100,0 $\%$ ) y en los universitarios de $12,9 \%$ (4,3-21,4 \%). Para la provincia Granma la tasa de discapacidad física en los analfabetos fue de 55,6\% $(48,2-62,9 \%)$ y en los universitarios de $25,0 \%(10,0-40,0 \%)$. En Holguín los analfabetos mostraron una tasa de $43,8 \%(27,4-60,2 \%)$ y los universitarios de $0 \%$.

Aunque la tendencia en todas las provincias fue hacia la reducción de la discapacidad con el incremento de la escolaridad, la imprecisión de algunos intervalos de confianza, limita la validez de estos resultados.

\section{DISCUSI ÓN}

La definición de discapacidad física que se utilizó en este estudio, de que el individuo no sea capaz de realizar al menos una AIVD, es la empleada en la mayoría de los estudios que abordan esta problemática. ${ }^{14}$

El siglo XX presentó cambios dramáticos en los tipos de enfermedades que más afectan la salud de la población. En la actualidad, la discapacidad entre las personas mayores es causada principalmente por enfermedades no transmisibles. Hay autores que plantean que la discapacidad no es un atributo que está claramente presente o ausente en la persona, sino que puede tener diversos grados de gravedad.

En el presente trabajo, la prevalencia de discapacidad física que se registró para las provincias que participaron en esta investigación, con excepción de Holguín, fue superior a la encontrada por Bayarre. ${ }^{1}$ Tello ${ }^{12}$ refiere una prevalencia superior a la encontrada en esta investigación. Todos estos estudios utilizaron la misma metodología y aplicaron los mismos instrumentos.

Investigaciones anteriores realizadas en otras latitudes, en la década de los 80 , como las de Bond y otros ${ }^{8}$ informan una prevalencia de discapacidad física en el 
anciano inferior a la encontrada en cuatro de las provincias investigadas, y muy parecida a la de Holguín. Sin embargo, estos consideran como discapacitado al anciano que no pueda realizar tres o más AIVD lo que pudiera explicar la diferencia entre las prevalencias registradas y, al mismo tiempo, limita la comparación.

Gorgocena y Algarin (1987), citado por Bayarre ${ }^{1}$ estiman una prevalencia de discapacidad física, en un área de salud del municipio Playa, similar a la de Holguín, e inferior a la encontrada en las restantes provincias y los demás estudios realizados en Cuba, pero esta investigación no se realizó con un adecuado diseño de la muestra, lo que pudiera influir en los resultados.

Pardavilla ${ }^{10}$ en España, toma como criterio de discapacidad física en el anciano la incapacidad para poder realizar al menos una de las AVD, encuentra una cifra ligeramente superior a la estimada para Holguín e inferior a la de las restantes provincias estudiadas en la presente investigación.

Beland y Zunzunegui ${ }^{15}$ realizaron un estudio de discapacidad en ancianos españoles y encuentran que la mitad de los encuestados tiene limitación para al menos una de las AIVD. Más recientemente, Nourhanshemi ${ }^{16}$ registra una cifra de discapacidad física similar a la encontrada para la mayoría de las provincias de este estudio.

Varios autores han señalado que la disimilitud de los resultados notificados por distintas investigaciones puede deberse, entre otras razones al modo de aplicación de los instrumentos, - la discapacidad referida por autorreporte es generalmente inferior a la obtenida por informantes-, la propia definición de discapacidad y las características del instrumento utilizado. Desde luego que estos aspectos, devienen fuente de sesgos para la medición de este evento, por lo que limita la comparación entre los resultados de diferentes investigaciones. ${ }^{17}$

Más allá de los efectos biológicos del envejecimiento, muchos de los posibles factores de riesgo de las discapacidades comienzan a presentarse a partir de la quinta década de la vida. ${ }^{18} \mathrm{El}$ aumento del riesgo de discapacidad física encontrado en esta investigación, en la mayoría de las provincias estudiadas, respecto a otros reportes, puede relacionarse con la crisis económica que ha afectado al país desde la década de los años 90, con repercusiones importantes sobre el estado de salud de los ancianos. Asimismo, el perfeccionamiento de los diseños metodológicos de estas investigaciones pudiera haber contribuido a una mejor estimación de la verdadera prevalencia de la discapacidad física, lo que traería como consecuencia el aumento en el valor estimado.

Por otra parte, como se vio en el apartado anterior, la escasa diferencia en los niveles de discapacidad física encontrados entre las poblaciones geriátricas de la Ciudad de La Habana, Camagüey, Las Tunas y Granma se relaciona con las diferencias en las estructuras por edad de estas (tabla 2). A ello es probable que se le adicione el comportamiento de otras variables no controladas con el procedimiento utilizado, como estilos de vida diferentes en las poblaciones en estudio.

La realización de las AIVD se comportó de forma similar a lo informado por estudios epidemiológicos realizados en varios países desarrollados, donde se ha observado más discapacidad para aquellas actividades que requieren mayor esfuerzo físico y desplazamiento del anciano. ${ }^{1}$ En Cuba, el aumento de la dependencia del anciano para realizar las actividades que requieren un mayor desplazamiento, probablemente está relacionada con las limitaciones en la disponibilidad del transporte, recrudecidas en el llamado "período especial", lo que conlleva a que el 
anciano tienda a disminuir o eliminar esta actividad. Estos resultados coinciden con los encontrados por Tello. ${ }^{12}$

Zayas, según refiere Bayarre, ${ }^{1}$ en una investigación en el área de salud del policlínico "Cristóbal Labra" (1992), registra el modo de transporte como una de las actividades más afectadas, resultado con el que coincide este estudio, así como que la capacidad para manejar las finanzas fue la menos perjudicada. Sin embargo, esta última actividad fue la más afectada en los adultos mayores de las provincias Ciudad de La Habana y Camagüey, con tasas de prevalencia muy superiores a la encontrada por dicho investigador y por otros, ${ }^{1,12}$ lo que pudiera estar dado porque la población objeto de investigación en estas provincias tenía una edad promedio mayor, lo que conlleva una mayor frecuencia de limitaciones en la visión, audición, memoria, cálculo y atención, entre otras funciones, que pudieran dificultar el manejo del dinero. A esto se adiciona la circulación de una doble moneda, con tasas de cambio variables, lo que dificulta su manejo, sobre todo para los ancianos con baja escolaridad.

La discapacidad física según la edad se comportó de manera similar a lo informado en otros estudios. ${ }^{1,12,19}$ Ania Lafuente ${ }^{20}$ en un estudio en la población anciana de Canarias observa que hasta los 75 años las dos terceras partes de los ancianos son capaces para todas las AIVD, pero que esta capacidad disminuye a la mitad entre los 75 y 79 años, y más aún a partir de los 85 años de edad.

Los estudios citados refieren que a medida que transcurren y se acumulan los años, varía la forma y el funcionamiento del cuerpo humano, el llamado envejecimiento normal. La disminución de la fortaleza muscular se ha asociado a mayores grados de discapacidad física. ${ }^{21} \mathrm{Si}$ bien las funciones fisiológicas se afectan con el proceso de envejecimiento, la mayoría de las personas ancianas no tienen alteraciones que influyan marcadamente en su funcionamiento físico, intelectual o social antes de los 80 años. Si se tiene en cuenta que este grupo de edad es el que más crecerá en las próximas décadas, se puede vaticinar un incremento marcado de esta problemática, lo que debe tomarse en cuenta por los que deciden políticas de salud, en la búsqueda de una longevidad más saludable.

En este estudio existió un predominio del riesgo de discapacidad en el sexo femenino al igual que en otras investigaciones con el mismo criterio de evaluación de la discapacidad. ${ }^{1,12,22}$ En 1992 Bond $^{8}$ y más recientemente otros investigadores ${ }^{23,24}$ notifican mayor riesgo en el sexo femenino, resultado con el que coinciden otras investigaciones cubanas. ${ }^{1,12}$

La diferencia de discapacidad física por sexo pudiera deberse a la discreta mejoría alcanzada en la esperanza de vida para el sexo femenino en relación con el masculino, lo que provoca un número mayor de efectivos poblacionales femeninos en los grupos de edad superiores, donde la discapacidad es más frecuente. Además puede estar relacionado con causas estrictamente biológicas debido a que existen enfermedades dependientes de la edad como Alzheimer, depresión, osteoporosis, artrosis y fractura de cadera, entre otras, altamente discapacitantes y las féminas son más vulnerables a ellas. ${ }^{19}$ Por otra parte, la presencia de un gran número de familias multigeneracionales en la población cubana, puede acarrear, bien la suplantación de las ancianas por las mujeres jóvenes de la casa en las labores domésticas, o por el contrario la sobrecarga. Estos cambios en el rol doméstico de las ancianas pudieran relacionarse con la diferencia encontrada. ${ }^{1}$

En relación con las enfermedades crónicas, los resultados fueron superiores a los de otros estudios. ${ }^{1,12}$ Las enfermedades crónicas, fundamentalmente las degenerativas 
del sistema osteomioarticular y algunas del sistema nervioso central, dificultan el desempeño de las actividades diarias del senescente.

La prevalencia de discapacidad física por secuelas posfractura de cadera fue similar a la obtenida por Tello. ${ }^{12}$ Estos resultados están en concordancia con lo esperado, si se tiene en cuenta las serias limitaciones que estas fracturas producen y las alteraciones que provocan en las esferas psicológica y social del anciano. Sin embargo, otra investigación ${ }^{1}$ informa un riesgo inferior; en ella se considera que los resultados se deben a la gran letalidad que tiene este evento de salud en la población anciana y no a que el mismo provoque pocas limitaciones en las AIVD.

Con respecto a la pérdida del rol social, otros investigadores ${ }^{12}$ informan una tasa de discapacidad física mayor que la de este estudio. Algunos autores cubanos ${ }^{1,12}$ han planteado que la sociedad no ha precisado cuáles roles ha de desempeñar el adulto mayor. Asimismo se argumenta que la falta de labores específicas ocasiona la dificultad de no saber dónde concentrar los esfuerzos y dónde volcarse para actualizar sus propias potencialidades. De ahí que, como las actividades de los ancianos en la sociedad no han sido definidas en el nivel sociocultural, deban ser especificadas para cada sujeto en particular, de manera que cada anciano debe buscar qué hacer y crear sus propias tareas que pueden ser más o menos valiosas para él, pero sin la garantía de reconocimiento social.

Molinero ${ }^{25}$ en España ha descrito que el desarrollo industrial y de las instituciones han incidido en el campo de la educación y en la forma de comportamiento, en definitiva, en la escala de valores culturales y sociales donde el perfil de los mayores y su situación social ha experimentado profundos cambios en las últimas décadas y hasta se puede decir que han perdido su estatus y rol social.

En relación con la insatisfacción con las actividades cotidianas, una investigación previa $^{1}$ y Tello ${ }^{12}$ obtienen resultados inferiores a los de este estudio. El grado de insatisfacción pudiera estar muy ligado al fenómeno de la rutina, entendida como la reiteración no creativa de las actividades a lo largo de las semanas, meses y años. El nivel de insatisfacción con las actividades cotidianas puede estar influenciado por el hecho de que existen muchos ancianos jubilados que desempeñan actividades en la actualidad muy diferentes a las que desempeñaron en los años previos y para las cuales no se preparan adecuadamente desde el punto de vista emocional.

La ausencia de confidentes, con la excepción de Holguín, fue similar a lo encontrado por Tello, ${ }^{12}$ pero mayor que la de otros estudios. ${ }^{1,12,24}$ Llegada la senectud, las pérdidas personales, familiares, lo lento de la vida diaria, las limitaciones en la capacidad de funcionamiento autónomo, la fragilidad y el deterioro físico, provocan que el anciano se encierre en sí mismo y no pueda o no quiera compartir sus secretos con los que lo rodean en la comunidad o con convivientes más allegados.

Con la ancianidad se supone que un grupo de personas pase de la autonomía a depender de los demás, ${ }^{26}$ a lo que se le agrega la pérdida de las relaciones que han tenido durante años, tales como el trabajo, y sobre todo, los hijos. Estos ya pueden valerse por sí mismos, no necesitan de la ayuda de los padres, que tienden entonces a sentirse menos valiosos.

La sociedad ve la desocupación en el anciano como un hecho normal. ${ }^{1}$ En este estudio se obtuvo una tasa de discapacidad física según dicha variable inferior a las que precisan otras investigaciones ${ }^{12}$ con excepción de un estudio anterior realizado en el municipio Playa por Bayarre y otros. ${ }^{1}$ 
Con la jubilación los ancianos se acogen a un descanso merecido, después de muchos años de trabajo. Además, en Cuba fueron pocas las mujeres que en edades productivas trabajaron fuera de sus casas, debido a que por normas de la sociedad de índole sociocultural, las mujeres debían permanecer en sus hogares, desempeñando tareas domésticas y dependiendo económicamente de sus esposos.

Los resultados obtenidos con respecto a los sentimientos de soledad y la discapacidad física mostró una prevalencia superior en Ciudad de La Habana con respecto a las restantes provincias, y a lo informado por otros autores. ${ }^{1,12}$ Esto pudiera relacionarse con el carácter cosmopolita de la capital, que genera una vida más convulsa, sujeta a un ritmo más intenso y el que tiende a producir la llamada "intimidad a distancia", o sea, a que el anciano se sienta solo aún cuando físicamente este rodeado de familiares.

En los ancianos predominaron los sentimientos de soledad, los cuales se acentuaron con la pérdida de seres queridos, la jubilación, la inactividad y la incomunicación de las jóvenes generaciones con las viejas al creer que los ancianos por su condición y poco aporte a la sociedad son inútiles, lo que favoreció su aislamiento y que se desarrollara ese sentimiento en ellos.

La prevalencia de discapacidad física por las inadecuadas condiciones de vida, no se diferenció de forma importante entre la mayoría de los territorios investigados. Se exceptuó la provincia Holguín, para la que se registró una tasa muy inferior. Tello ${ }^{12}$ obtiene una tasa de discapacidad física según esta variable superior a las encontradas aquí y a las de estudios anteriores.

A pesar de que la seguridad social desempeña un papel importante en las condiciones económicas de los ancianos, es aún insuficiente para satisfacer algunas de sus necesidades materiales. ${ }^{1,15}$ Las condiciones de las viviendas cubanas, a raíz del "período especial", han venido sufriendo un paulatino deterioro, por la escasez de recursos para su mantenimiento; tal situación incide negativamente en la población en general y en los ancianos en especial.

La presencia de pérdidas relacionada con discapacidad mostró una prevalencia superior a la encontrada en estudios anteriores. ${ }^{1,12}$ Durante la vejez se acumulan numerosas pérdidas significativas: seres queridos, salud física, autonomía funcional, independencia económica, así como, cambios de rol, tanto familiar como social; pérdidas y cambios que obligan a una reestructuración considerable que permita la adaptación a las nuevas condiciones, lo que puede conllevar a la posibilidad de fracasar en dichos mecanismos. ${ }^{26}$

En cuanto a la inadaptación, a la jubilación y la discapacidad física, se obtuvo una tasa de prevalencia superior a otros reportes, excepto Holguín que tuvo una tasa de cero. ${ }^{1,12}$ Los argumentos expuestos en relación con la sensación de inactividad pudieran explicar las diferencias encontradas en esta tasa entre los territorios estudiados.

Los resultados obtenidos con las variables psicosociales relacionadas con la discapacidad pudieran estar relacionados con la existencia de múltiples factores que incidieron negativamente en la calidad de vida del anciano, por lo que se requiere de un enfoque multidisciplinario e intersectorial en la atención a este grupo de edad.

El comportamiento de la discapacidad física según la escolaridad coincidió de forma general con otros autores, ${ }^{1,12,27}$ los que informan que a menor escolaridad hay mayor discapacidad. Estos resultados reflejan que el nivel educacional contribuye al 
bienestar de los ancianos, al posibilitar que las personas se preparen mejor para su vejez.

La discapacidad física afectó, en general, a un tercio de la población de estudio, con diferenciales entre provincias, que ubicaron a Holguín como la de menor afectación, en tanto Granma fue la más comprometida. La discapacidad se incrementa con la edad, predomina en el sexo femenino, y está generada fundamentalmente por pérdida de actividades que demandan mayor desplazamiento del anciano. Las categorías de las variables psicosociales más afectadas en general son: analfabetismo y pérdida de rol social, con excepción de Holguín, en la que las mayores afectaciones ocurren con la sensación de inactividad y la insatisfacción con las actividades cotidianas. En Ciudad de La Habana y Granma se adicionaron los sentimientos de soledad, en Camagüey la insatisfacción con actividades cotidianas y en Las Tunas la sensación de inactividad.

\section{REFERENCI AS BI BLI OGRÁFICAS}

1. Bayarre Vea H, Fernández Fente A, Trujillo Gras O, Menéndez J iménez J . Prevalencia de discapacidad física en ancianos del municipio Playa. 1996. Parte I. Rev Cubana Salud Pública. 1999; 25 (1):16-29.

2. Hernández Castellón R. Estudio del envejecimiento de la población. En: CEDEM, editor. Perspectivas y escenarios de la población y los recursos humanos de Cuba y sus implicaciones económicas y sociales entre el año 2000 y 2050. La Habana: CEDEM; 2000.p.374-418.

3. Bayarre Vea H, Fernández Fente A, Trujillo Gras O, Menéndez J iménez J . Prevalencia de discapacidad mental en ancianos del municipio Playa. 1996 Parte II. Rev Cubana Salud Pública. 1999; 25 (1):30-8.

4. Fillembaum GG. Assessing wellbeing to the elderly. Adv Res. 1984; 8: 7-11.

5. Patterson RL, Eberly DA. The modular approach to behavior modification with the elderly. En: Patterson, editor. Overcoming deficits of aging: a behavioral approach. New York: Patterson; 1982. p.53-87.

6. Katz SA. Akpon CA. A measure of primary sociobiological characteristics. J Term J Health Service. 1986:493-507.

7. Grieco M, Apt N. El desarrollo y el envejecimiento de la población. Síntesis mundial por expertos en el envejecimiento en África En: ONU, editor. La Situación del envejecimiento de la población mundial. Hacia una sociedad para todas las edades. Nueva York: Naciones Unidas;2001.p.14-42.

8. Bond J, Constairs V. Services of the elderly Scottish Health Services. Studies No. 42, Edimburg: S/E; 1999.

9. Pearlman RA, Utilman RF. Quality of life in chronic diseases: perceptions of elderly patients. J Gerontol. 1998;43:25-30.

10. Pardavilla B. Aproximación a las necesidades de ayuda a domicilio y plazas residenciales en la población española mayor de 65 años. Rev Serv Social Pol Sociales. 1989; 14: 28-37. 
11. Fiallo A. Hacia la caracterización del anciano cubano en un área de salud. Rev Hosp Psiq Habana. 1992; 33: 23-7.

12. Tello Velázquez Y, Bayarre Vea H, Herrera Domínguez H, Hernández Pérez Y. Prevalencia y factores de riesgo de discapacidad física en ancianos. Municipios Amancio Rodríaguez y J obabo. 1999. Rev Cubana Salud Pública. 2001;27(1): 19-25.

13. Silva Ayçaguer LC. Diseño razonado de muestras y captación de datos para la investigación sanitaria. Madrid: Díaz de Santos; 2000.

14. Palmore E, Burchett B. Predictors of disability in the final years of live. J Aging Health. 1997; 9:283-98.

15. Beland F, Zunzunegui MV. Predictors of functional status in older people living at home. Age-Ageing. 1999; 28: 153-9.

16. Nourhashemi F, Andrieu S, Gillette-Guyonnet S, Vellas B, Albarede J L, Grandjean $\mathrm{H}$. Instrumental activities of daily living as a potential marker of frailty: a study of 7364 community-dwelling elderly women (the EPIDOS study). J Gerontol A Biol Sci Med Sci. 2001;56(7): M448-53.

17. Rodgers W, Miller B. A comparative analysis of ADL questions in surveys of older people. J Gerontol, Serie B. 1997; 52B (Special Issue):21-36.

18. Reed D, Foley D, Wuite L, Heimovitz H, Burchfiel C, Masaki K. Predictors of healthy aging in men with high life expectancies. Am J Public Health.

1999; 88: 1463-8.

19. Guralnik E. Physical disability in elder Americans. J Gerontol. 1998;48(Especial Issue): 3-10.

20. Lafuente A. Vejez saludable e incapacidad funcional en la población anciana de Canarias. Rev Española Salud Pública. 1997;2:14-21.

21. Rantanen T, Guralnik J, Sakary-Rantala R, Leveille S, Simonsick E, Ling S, et al. Disability, physical activity and muscle strength in older women: the women's health and aging study. Arch Phys Med Rehabil. 1999; 80: 130-5.

22. Schroll Bjornsbo K, Ferry M, de Groot CP, Schlienger J L. Changes in physical performance in elderly Europeans. 2002. SENECA. 1993-1999;6(1):9-14.

23. Melzer D. Profile of disability in elderly people: estimates from a longitudinal population study. BMJ . 1999; (318): 1108-12.

24. March LM, Brnabic AJ, Skinner JC, Schwarz JM, Finnegan T. Musculoskeletal disability among elderly people in the community. Med J Aust. 1998; (168):439-42.

25. Molinero S. Fragilidad en la esfera social. Rev Esp Geriatr Gerontol. 1997; 32: 21-5.

26. Bárcena A, Sánchez MI. Fragilidad afectiva en el anciano. Rev Esp Geriatr Gerontol. 1997; 32: 15-20. 
27. Melzer D, Izmirlian G, Leveille SG, Guralnik J M. Educational differences in the prevalence of mobility disability in old age: the dynamics of incidence, mortality, and recovery. J Gerontol B Psychol Sci Soc Sci. 2001;56(5):S294-301.

Recibido: 20 de noviembre de 2007.

Aprobado: 14 de diciembre de 2007.

Héctor Bayarre Vea. Calle 80 No. 1509 e/ 15 y 17. Playa. La Habana, Cuba. Email: hbayarre@infomed.sld.cu, hdbayarre@yahoo.com

Tabla 1. Prevalencia de discapacidad según provincia

\begin{tabular}{|c|c|c|c|}
\hline \multicolumn{1}{|c|}{ Provincia (n) } & No. & Tasa $^{*}$ & IC (95\%) \\
\hline \begin{tabular}{c|c|c|} 
Ciudad de La.Habana \\
(728)
\end{tabular} & 253 & 34,8 & $32,6-37,0$ \\
\hline Camagüey (866) & 305 & 35,2 & $32,0-38,4$ \\
\hline Las Tunas (698) & 247 & 35,4 & $27,9-42,8$ \\
\hline Granma (688) & 252 & 36,6 & $31,6-41,7$ \\
\hline Holguín (695) & 96 & 13,9 & $8,5-19,3$ \\
\hline
\end{tabular}

n: número de ancianos, *por 100 adultos mayores.

Tabla 2. Tasas de discapacidad física (TDT)* tipificadas por edad y sexo según provincia

\begin{tabular}{|c|c|c|}
\hline Provincia & TDT edad & TDT sero \\
\hline Ciudad de La Habana & 24,61 & 33,92 \\
\hline Camagüey & 36,37 & 34,35 \\
\hline Las Tunas & 34,84 & 38,99 \\
\hline Granma & 36,84 & 35,41 \\
\hline Holguín & 13,66 & 14,88 \\
\hline
\end{tabular}

*por 100 adultos mayores 
Tabla 3. Prevalencia de discapacidad física en ancianos según variables biológicas y provincia

\begin{tabular}{|c|c|c|c|c|c|c|}
\hline \multirow[t]{3}{*}{ Provincia } & \multicolumn{6}{|c|}{ Variables biológicas } \\
\hline & \multicolumn{3}{|c|}{ Enfermedades crónicas } & \multicolumn{3}{|c|}{$\begin{array}{c}\text { Secuela posfractura de } \\
\text { cadera }\end{array}$} \\
\hline & No, & Tasa* $^{*}$ & IC $(95 \%)$ & No, & Tasa* $^{*}$ & IC $(95 \%)$ \\
\hline $\begin{array}{l}\text { Ciudad de } \\
\text { La Habana }\end{array}$ & 184 & 41,6 & $39,6-43,6$ & 7 & 70,0 & $44,5-95,6$ \\
\hline Camagüey & 142 & 29,8 & $25,7-33,9$ & 21 & 77,8 & $62,1-93,5$ \\
\hline Las Tunas & 151 & 32,5 & $20,4-44,6$ & 8 & 80,0 & $46,2-100,0$ \\
\hline Granma & 152 & 40,8 & $34,6-47,0$ & 6 & 66,7 & $46,7-86,7$ \\
\hline Holguín & 52 & 13,9 & $11,9-15,9$ & 4 & 100,0 & - \\
\hline
\end{tabular}

* por 100 adultos mayores, Su cálculo se realizó tomando como denominador el total de ancianos de cada variable biológica y provincia, 